Lastly, it may be pointed out in such a discussion as this that it is quite justifiable to suggest general points of interest, although in similar discussions in all congresses the same questions recur and have been dealt with. We are all aware that no subject offers such an opportunity for anecdote and humour, but, setting this aside, many important questions suggest themselves, such as the mental effects upon patients who imagine they are suffering from the effects of foreign bodies and the great variety of bodies which find their way into air-passages. The all-important questions of early detection, the duration which a body may rest within the air-passages followed by slight or serious effects, methods of removal, the sequelæ, feeding of patients while the foreign body remains in the air-passages, relief to breathing, and treatment of the spasms where dyspncea is threatened, imperfect or careless function in swallowing, are all worthy of your consideration. In mentioning such points one is only too conscious of the fact that it is only possible in opening such a discussion to rapidly indicate points of value for profitable consideration.

Speaking generally, I have only seen one case which proved fatal, and that was in an infant brought to me with cellulitis of the neck, and where several unsuccessful attempts had previously been made to remove the foreign body. In only one case, and that not in my own practice, but in that of one of my colleagues, have I seen it necessary to advise opening the trachea. In this case severe inflammation of the lungs with threatening dyspnoa necessitated this procedure, and the patient made an excellent recovery a few days after the foreign body had been coughed up to the tracheal opening, when it was easily extracted.

\title{
THE CONNECTION OF THE ISOLATED RESPIRATORY FIBRES OF THE RECURRENT WITH THE SYMPATHETIC AND CARDIAC NERVES.
}

\author{
By Adolf Onodi, M.D., \\ Professor of Khino-Laryngology, University of Buda-Pesth.
}

THe connections of the laryngeal nerves with the sympathetic and the cardiac nerves have been known for a long time, but recent observations and my investigations have shown an extraordinarily intimate connection, which doubtless must have special significance. The connection of the superior laryngeal nerve with the upper cervical ganglion of the sympathetic and with the superior cardiac 
branches which arise from the latter is known, as is also the connection of the superior laryngeal nerve with the lower cervical ganglion of the sympathetic, the connection of the external branch of the superior laryngeal being much closer with the upper cervical ganglion of the sympathetic and the superior cardiac nerve.

The external branch of the upper laryngeal nerve sends off a direct cardiac branch or connections to the upper cervical ganglion of the sympathetic and to the upper cardiac nerve. These anatomical conditions form in man an exact analogue to the depressor nerve found in animals. In regard to the inferior laryngeal, we know its connections with the upper cervical ganglion of the sympathetic and with the superior cardiac nerve. Of great signiticance is the observation of Lenhossék, according to which the inferior laryngeal nerve arises from a ganglion, whilst the ganglion stands in connection with the pneumogastric by means of a thin root. The lower laryngeal is connected with the lower cervical ganglion of the sympathetic, and further with the cardiac branches, which take their origin from the middle and lower cervical sympathetic.

I have succeeded in giving in the horse the anatomical proof that, according to the double function of respiration and phonation of the larynx, the respiratory and phonatory nerve bundles run separately in the trunk of the inferior laryngeal nerve and in the trunk of the pneumogastric. I have demonstrated this for a length of 88 centimetres. Whilst the phonatory nerve bundle could easily be isolated, and showed only one connection each with the ansa Vieussensii and with the cardiac branch, it was difficult to isolate the respiratory nerve bundle, for it was very intimately connected by means of eight branches with the sympathetic and the cardiac branches. [The photograph exhibited showed this remarkable intimate connection.]

It is known that in the trunks of the sympathetic, besides the vasomotor and trophic, there are cerebro-spinal, sensitive, and motor fibres. My investigations have shown that the cerebrospinal nerve fibres of the rami communicantes run in the trunk of the sympathetic according to a well-defined system. In the upper part of the thorax and in the neck the larger part of the cerebrospinal fibres of the sympathetic run upwards, and only a smaller part downwards, whilst in the rest of the thorax and in the abdomen just the reverse is the case. The sympathetic constitutes the morphological basis in the known connections for the course of the fibres and their intercommunication, as they are of different origin and different destination. Physiology has until now shown the presence of the depressor fibres and the cardiac branches 
originating from the upper laryngeal nerve, and my experiments have shown that in the dog the irritation of the sympathetic trunk between the lower cervical and first thoracic ganglion, as also of the communicating branches of the brachial plexus, will produce a contraction of the vocal cords of the same side. Beyond these observations the morphological facts mentioned have received no pathological or physiological explanation. I may, in particular, draw attention to the intimate connection before mentioned which exists between the isolated respiratory nerve fibres of the lower laryngeal nerve and the sympathetic and the cardiac branches. The morphological foundation is laid in the above-mentioned facts, showing an exchange of nerve fibres of different origin and destination, but the isolation of these fibres and the investigation of their destinations forms still a subject the difficult solution of which is reserved for future physiological and pathological inquiry.

\section{A SIMPLIFIED METHOD OF OPERATING FOR DEFLECTION OF THE CARTILAGINOUS SEPTUII.}

$$
\text { Bi Dundas Grant, M.D., F.R.C.S. }
$$

Since the publication of the description of Moure's operation for deflected septum in the Journil of Laringologr, ${ }^{1}$ it would be strange if those rhinologists who read it had failed to put it in practice, as its principles were bound to appeal to those who had experienced difficulties in the operations previously recommended for its purpose. I have used it in three cases with excellent results.

It will be remembered that, after the shaving off of any prominent thickening ot the cartilaginous septum, the mucous membrane is allowed to heal up, and then-say at an interval of three or four weeks-two cuts are made in the septum, one obliquely upwards and backwards, parallel to the ridge of the nose, the other horizontally, near and parallel to the floor. A somewhat tapering tube, one side of which is made of a slip of soft metal, is introduced into the narrow nostril by means of a pair of expanding forceps; these forceps are then opened so as to press the scptum across into the opposite nostril, the tube is left in situ for a week. and is then removed. In my cases it has caused little or no irritation, has not usually got plugged with clot, and has offered no obstacle to respiration. In some cases nothing further is required, but in others there may be thickenings above and below the position of the tube, which may afterwards be easily diminished by means of the knife.

Of all the methods devised for meeting the difficulty of making a straightened-out septum find room between the fixed points at the

$$
\text { I Vol. xvi., p. } 163 .
$$

